\title{
Severe acute exacerbation of chronic hepatitis B during pegylated interferon treatment and early intervention with corticosteroid
}

\author{
Qing Mao, Hui-Yan Zhang, Jian-Ping You and Xu-Qing Zhang*
}

\begin{abstract}
Severe acute exacerbation or liver failure induced by standard interferon-a(IFN-a) therapy had been reported to occur in few patients with chronic hepatitis B. However, no report showed that pegylated interferon-a therapy was able to induce severe acute exacerbation of chronic hepatitis B. Here, we describe three patients with severe acute exacerbation of chronic hepatitis B during pegylated interferon-a2a (Pegasys) treatment. One patient progressed into acute-on-chronic liver failure (ACLF) at the second week of Pegasys treatment. Two patients progressed into acute-on-chronic pre-liver failure (pre-ACLF) at the second and eighth week of Pegasys treatment, respectively. Three patients recovered after early combined intervention with corticosteroid and lamivudine. Our data indicated that there was a risk of severe acute exacerbation among patients with chronic hepatitis B during receiving Pegasys treatment. Importantly, early combined intervention with corticosteroid and lamivudine should be introduced to prevent the disease progression and improve their prognosis once severe acute exacerbation was diagnosed.
\end{abstract}

Keywords: Chronic hepatitis B, Acute-on-chronic liver failure, Severe acute exacerbation, Corticosteroid, Pegylated interferon

\section{Introduction}

It is estimated that over 350 million people worldwide are chronically infected with hepatitis B virus (HBV). Chronic hepatitis B is one of the most important causes of cirrhosis and hepatocellular carcinoma [1]. Injected interferons (standard interferon- $\alpha$ and pegylated interferon- $\alpha$ ) are approved to treat chronic hepatitis B in many countries, and have been confirmed to be effective in preventing the disease progression of chronic hepatitis B. Acute exacerbation of chronic hepatitis B is not uncommon during interferon- $\alpha(\mathrm{IFN}-\alpha)$ therapy because that IFN- $\alpha$ exerts a variety of immuno-modulatory effects [2]. However, there were very few reports about severe acute exacerbation of chronic hepatitis B during IFN- $\alpha$ therapy [3,4]. From January 2008 to December 2010, 254 patients with chronic hepatitis B received Pegylated interferon- $\alpha 2 \mathrm{a}$ (Pegasys) therapy in our department. During Pegasys therapy, alanine aminotransferase (ALT) flare was observed in $25.2 \%(64 / 254)$ patients, including

\footnotetext{
* Correspondence: xuqing651005@tom.com

Department of Infectious Diseases, Southwest Hospital, Third Military Medical University, Chongqing 400038, People's Republic of China
}

9.4\% (24/254) patients with serum ALT $\geq 420$ IU/L (normal range $0-42 \mathrm{IU} / \mathrm{L}$ ) and serum total bilirubin (TBil) $\leq 51.3 \mu \mathrm{mol} / \mathrm{L}$ (normal range 6-17.1 $\mu \mathrm{mol} / \mathrm{L}$ ), and $1.2 \%(3 / 254)$ patients with severe acute exacerbation (serum ALT $\geq 420 \mathrm{IU} / \mathrm{L}$ and T-Bil $>51.3 \mu \mathrm{mol} / \mathrm{L}$ ). In addition, severe acute exacerbation of chronic hepatitis $B$ having a potential for progression to acute on chronic liver failure (ACLF) or acute on chronic pre-liver failure (preACLF) with extremely high mortality, so it is highly important how to prevent the disease progression $[5,6]$. Here, we introduced three patients with severe acute exacerbation of chronic hepatitis B during Pegasys treatment, including two patients with pre-ACLF and one patient with ACLF. Three patients were successfully treated with early intervention of corticosteroid and lamivudine.

\section{Case report}

\section{Patients}

Three chinese male patients were hospitalized in the Department of Infectious Diseases, Southwest Hospital, Third Military Medical University, China, between January 2009 and October 2010. Serologic tests for hepatitis A 
virus (HAV), hepatitis $\mathrm{C}$ virus (HCV), hepatitis D virus (HDV), hepatitis E virus (HEV),cytomegalovirus (CMV), Epstein-Barr virus (EBV), and human immunodeficiency virus (HIV), and immunologic tests for liver and kidney microsomal, mitochondrial, smooth muscle, and nuclear antibodies were negative in three patients before and after receiving Pegasys therapy. Three patients were excluded to have cirrhosis by abdominal ultrasound, abdominal computed tomography scan, and Fibroscan before receiving Pegasys therapy. They did not receive any drugs other than Pegasys or alcoholism before acute exacerbation of chronic hepatitis B. Clinical characteristics of three patients with chronic hepatitis $\mathrm{B}$ at the baseline of interferon treatment were shown in Table 1.

\section{Severe acute exacerbation of hepatitis B during pegasys treatment}

Severe acute exacerbation of chronic hepatitis B was observed in two patients at the second week of Pegasys

Table 1 Clinical characteristics at the baseline of Pegasys treatment

\begin{tabular}{|c|c|c|c|}
\hline & Case 1 & Case 2 & Case 3 \\
\hline Age (yr) & 23 & 54 & 42 \\
\hline Course of disease $(\mathrm{yr})$ & 6 & 2 & 17 \\
\hline HBV genotype & B & $\mathrm{C}$ & B \\
\hline HBeAg & + & - & - \\
\hline Anti-HBe & - & + & + \\
\hline HBV DNA (Lc/ml & 7.78 & 6.90 & 6.99 \\
\hline WBC $\left({ }^{x} 10 / L\right.$ & 7.5 & 5.89 & 5.69 \\
\hline $\mathrm{Hb}(g / L)$ & 156 & 137 & 152 \\
\hline $\mathrm{Plt}\left({ }^{\mathrm{x}} 10 / \mathrm{L}\right.$ & 135 & 112 & 101 \\
\hline ALT (IU/L) & 333 & 71 & 304 \\
\hline AST (IU/L) & 215 & 76 & 149 \\
\hline AST/ALT ratio & 0.65 & 1.07 & 0.49 \\
\hline ALP (IU/L) & 197 & 123 & 94 \\
\hline y-GTP(IU/L & 45 & 27 & 44 \\
\hline Total bilirubin(umol/L & 13.9 & 16.9 & 30.9 \\
\hline Prothrombin activity (\%) & 105 & 100 & 104 \\
\hline AFP (ng.ml) & 3.31 & 5.6 & 4.29 \\
\hline Creatinine $(\mu \mathrm{mol} / \mathrm{L})$ & 59 & 79 & 81 \\
\hline T3 free (pmol/L) & 4.1 & 3.9 & 3.6 \\
\hline T4 free (pmol/L) & 14.3 & 11.3 & 12.7 \\
\hline TSH (mlU/L & 2.1 & 1.2 & 1.4 \\
\hline Fibroscan (kPa) & 4.3 & 5.1 & 5.7 \\
\hline
\end{tabular}

yr: year; Course of disease: interval between the first time when HBsAgpositive was detected and the date before treatment; HBV: hepatitis B virus; HBeAg: hepatitis B e antigen; LC/ml: Log copies/ml; WBC: white blood cell; Hb: hemoglobin; Plt: platelet; ALT: alanine aminotransferase; AST: asparate aminotransferase; ALP: alkaline phosphatase; $\gamma$-GTP: gamma glutamil transpeptidase; AFP: alpha fetoprotein; T3 and T4: thyroxin 3 and 4; TSH: thyroid-stimulating hormone. treatment $(180 \mu \mathrm{g}$, one time per week), and in one patient at the eighth week of Pegasys treatment (180 $\mu \mathrm{g}$, one time per week). Pegasys treatment induced the development of ACLF in one patient, and pre-ACLF in two patients. Clinical characteristics at onset of severe acute exacerbation among three patients with chronic hepatitis B were shown in Table 2.

\section{Clinical course and treatment}

Case 1 was tested for serum ALT being 96 IU/L, T-Bil $11.4 \mu \mathrm{mol} / \mathrm{L}$ and HBV DNA $7.86 \log _{10}$ copies $/ \mathrm{ml}(\mathrm{LC} / \mathrm{ml})$ on December 11 of 2008, but did not receive any antiviral treatment. After one month (January 11, 2009), he was tested for serum ALT being $333 \mathrm{IU} / \mathrm{L}, \mathrm{T}-\mathrm{Bil} 13.9 \mu \mathrm{mol} / \mathrm{L}$ and HBV DNA $7.78 \mathrm{LC} / \mathrm{ml}$, and received antiviral treatment with Pegasys $(180 \mu \mathrm{g}$, one time per week). He only felt slight fever (body temperature $38.1^{\circ} \mathrm{C}$ ) and malaise at the first day of Pegasys treatment, and was not observed to be dark-coloured urine and icteric bulber conjunctiva during the first week Pegasys treatment. However, he

Table $\mathbf{2}$ Clinical characteristics at onset of severe acute exacerbation

\begin{tabular}{|c|c|c|c|}
\hline & Case 1 & Case 2 & Case 3 \\
\hline Weeks of pegasys trreatment & 2 & 8 & 2 \\
\hline $\mathrm{HBeAg}$ & + & - & - \\
\hline Anti-HBe & - & + & + \\
\hline HBV DNA (LC/ml) & 5.74 & 6.23 & 4.10 \\
\hline $\operatorname{WBC}\left(10^{9} / \mathrm{L}\right)$ & 3.82 & 2.95 & 4.24 \\
\hline $\mathrm{Hb}(\mathrm{g} / \mathrm{L})$ & 151 & 136 & 142 \\
\hline $\operatorname{Plt}\left({ }^{\mathrm{x}} 10^{9} / \mathrm{L}\right)$ & 85 & 55 & 64 \\
\hline ALT (IU/L) & 1672 & 419 & 566 \\
\hline AST (IU/L) & 1533 & 320 & 394 \\
\hline AST/ALT ratio & 0.92 & 0.76 & 0.70 \\
\hline ALP (IU/L) & 175 & 177 & 188 \\
\hline$y-G T P(I U / L)$ & 170 & 181 & 318 \\
\hline Total protein(g/L) & 57.7 & 70.6 & 60.0 \\
\hline Albumin (g/L) & 34.2 & 33.0 & 34.5 \\
\hline Total bilirubin( $\mu \mathrm{mol} / \mathrm{L})$ & 289.3 & 179.6 & 180.6 \\
\hline Direct bilirubin ( $\mu \mathrm{mol} / \mathrm{L})$ & 182.5 & 146.6 & 96.8 \\
\hline Prothrombin activity (\%) & 35 & 83.3 & 88.1 \\
\hline AFP (ng/ml) & 89 & 774.1 & 56.2 \\
\hline Creatine ( $\mu \mathrm{mol} / \mathrm{L})$ & 44.3 & 57 & 72.7 \\
\hline FT3 (pmol/L) & 3.6 & 3.2 & 3.8 \\
\hline FT4 (pmol/L) & 12.4 & 13.4 & 11.9 \\
\hline TSH (mIU/L) & 1.3 & 0.9 & 1.2 \\
\hline
\end{tabular}

HBeAg: hepatitis B e antigen; HBV: hepatitis B virus; LC/ml: Log copies/ml; WBC: white blood cell; Hb: hemoglobin; Plt: platelet; ALT: alanine aminotransferase; AST: asparate aminotransferase; ALP: alkaline phosphatase; Y-GTP: gamma glutamil transpeptidase; AFP: alpha fetoprotein; T3 and T4: thyroxin 3 and 4; TSH: thyroid-stimulating hormone. 
complained of reduced appetite, vomiting and darkcoloured urine, and his bulber conjunctiva was observed to be icteric at the eighth day of Pegasys treatment. So, he was admitted to our hospital, and diagnosed to be ACLF based on his laboratory findings at the eleventh day of Pegasys treatment (Table 2). After admission, he was immediately treated with dexamethasone (10 mg/d, intravenously, for five days) and lamivudine (LMV, $100 \mathrm{mg} / \mathrm{d}$ ). Serum ALT and T-Bil showed a decreasing tendency, and prothrombin activity (PTA) showed a increasing tendency, after which he started to receive a tapering-dose prednisolone therapy and antiviral treatment with LAM (Figure 1). The levels of serum ALT (35 IU/L), T-Bil $(14.1 \mu \mathrm{mol} / \mathrm{L})$ and PTA $(100 \%)$ were normalized at 12 weeks treatment, after which he stoped to receive prednisolone therapy and continued to take LAM. He had undetectable level of serum HBV DNA (Real-time polymerase chain reaction assay,Amplicor HBV Monitor Test, Roche Diagnostics, Mannheim, Germany; detection limit of $1 \times 10^{3}$ copies/ $\mathrm{mL}$ ) at 4 weeks LAM treatment, and sustained virologic response during LAM treatment. The loss of $\mathrm{HBeAg}$ was observed at 52 weeks LAM treatment, and HBeAg seroconversion at 64 weeks LAM treatment.

Case 2 started to complain of reduced appetite, headache, vomiting and dark-coloured urine after 8 weeks Pegasys treatment, and was admitted to our hospital. After admission, he was diagnosed to be pre-ACLF based on his laboratory findings (Table 2), and was immediately treated with dexamethasone $(10 \mathrm{mg} / \mathrm{d}$, intravenously, for five days) and LMV (100 mg/d). At 5 days of treatment, serum T-Bil was $81.4 \mu \mathrm{mol} / \mathrm{L}$, and the decline extent of serum T-Bil is $54.7 \%$, so dexamethasone therapy was stopped. After the cessation of dexamethasone therapy, the ALT and T-Bil still showed a decreasing tendency, and were normalized at 8 weeks treatment (Figure 2). He had undetectable level of serum HBV DNA at 8 weeks LAM treatment, and sustained virologic response during LAM treatment.

Case 3 was tested for serum ALT being 213 IU/L, TBil $30.2 \mu \mathrm{mol} / \mathrm{L}$ and HBV DNA 7.25 LC/ml on September 1 of 2010, and admitted to our hospital for antiviral therapy with Pegasys (180 $\mu$ g, one time per week) because of suffering from chronic hepatitis B on September 6 of 2010 (Table 1). The changes of ALT, T-Bil and PTA were shown in Figure 3. Because of serum T-Bil being $56.4 \mu \mathrm{mol} / \mathrm{L}$ and ALT being $549 \mu \mathrm{IU} / \mathrm{L}$ (normal $<42 \mathrm{IU} / \mathrm{L}$ ) at $13^{\text {th }}$ day of Pegasys treatment, he started to receive LAM therapy instead of Pegasys. However, serum T-Bil was still rapidly increased after 4 days LAM treatment. He was diagnosed to be ACLF based on his worsened laboratory findings (Table 2), and immediately received a tapering-dose corticosteroid therapy (Figure 3). After corticosteroid therapy, the ALT and T-Bil showed a decreasing tendency, and were normalized at 8 weeks treatment (Figure 3). He had

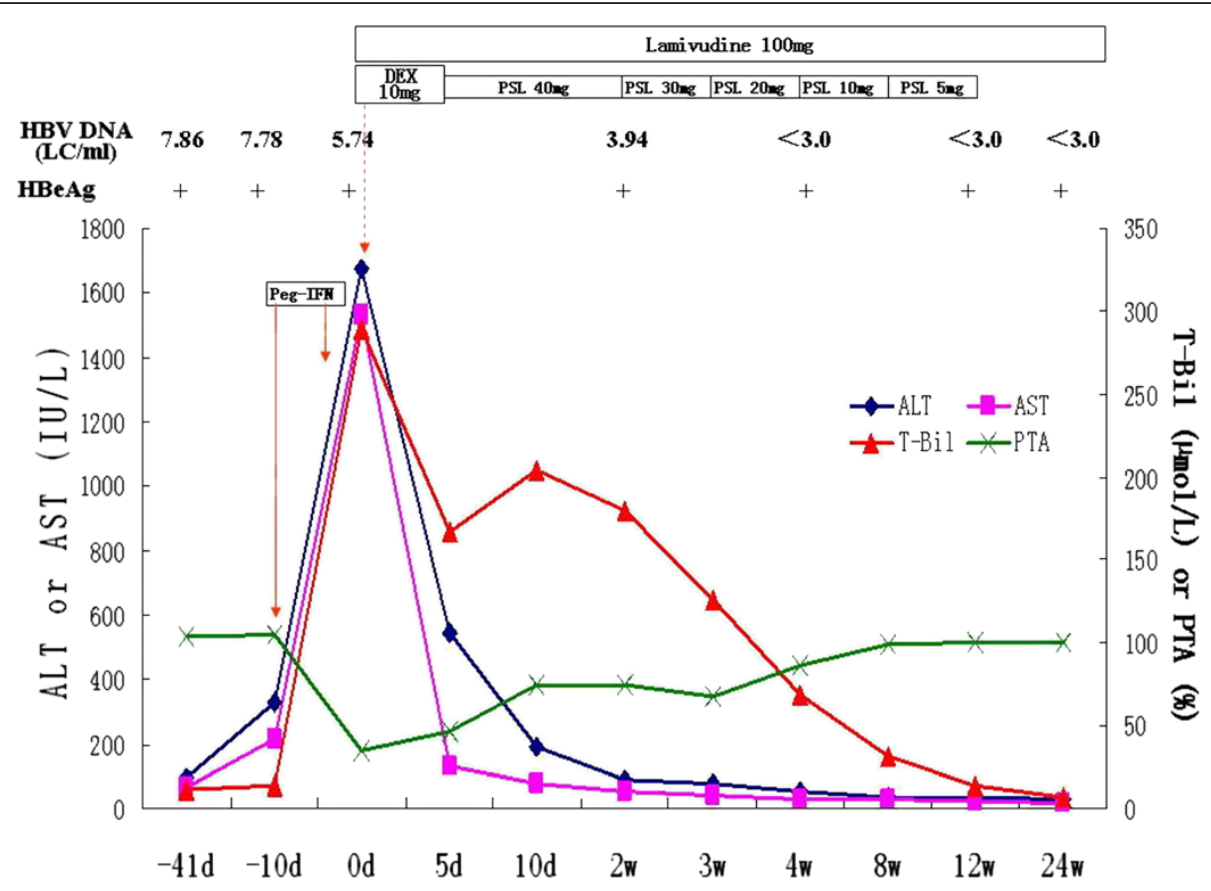

Figure 1 Clinical course of case 1. Peg-IFN: pegylated interferon-a2a; LC/ml: Log $_{10}$ copies/ml; DEX: dexamethasone; PSL: prednisolone; ALT: alanine aminotransferase; AST: asparate aminotransferase; T-Bil: total bilirubin; PTA: prothrombin activity. 


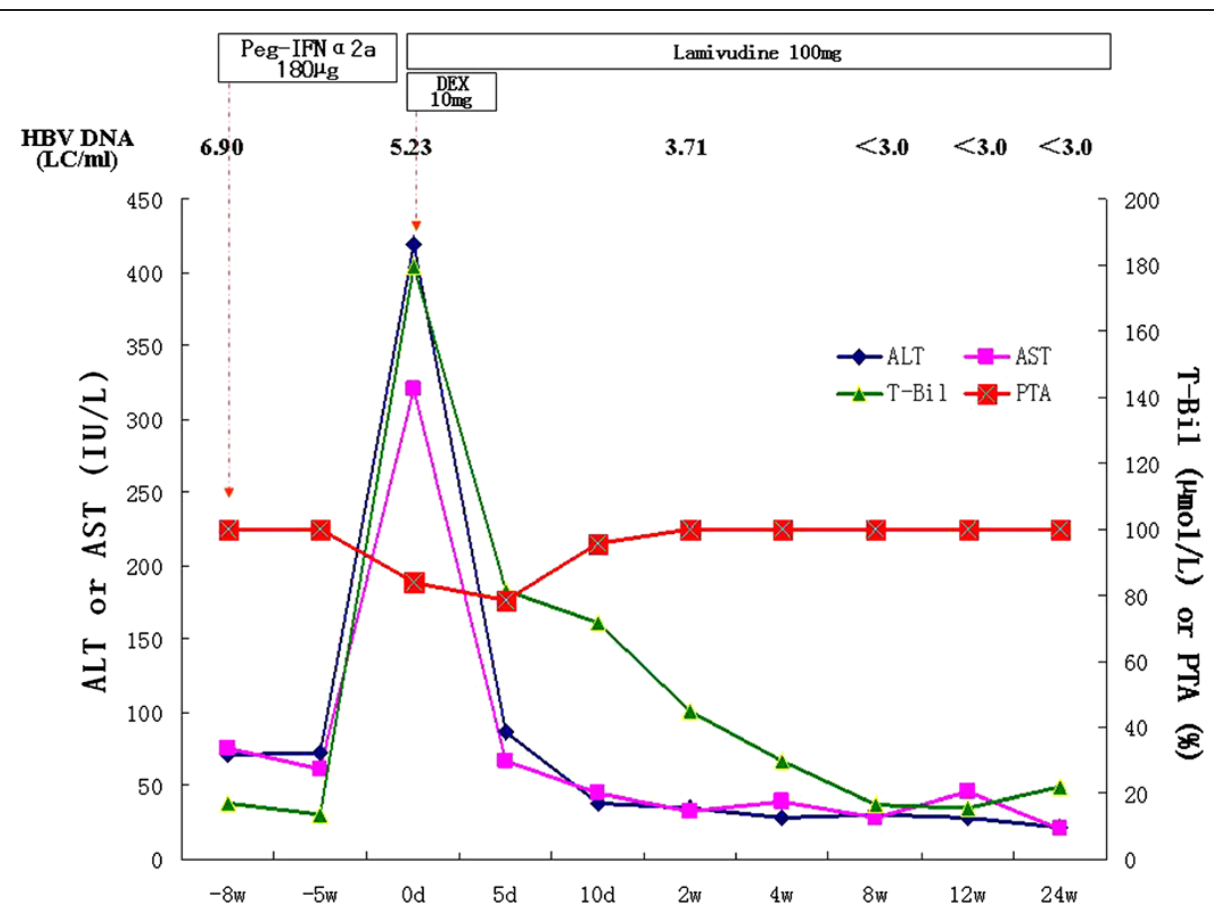

Figure 2 Clinical course of case 2. Peg-IFNa2a: pegylated interferon-a2a; LC/ml: $\log _{10}$ copies/ml; DEX: dexamethasone; ALT: alanine aminotransferase; AST: asparate aminotransferase; T-Bil: total bilirubin; PTA: prothrombin activity.

undetectable level of serum HBV DNA at 4 weeks LAM treatment, and sustained virologic response during LAM treatment.
Additionally, transfusion of magnesium isoglycyrrhizinate injection $(200 \mathrm{mg} / \mathrm{d})$ and reduced glutathione $(1200 \mathrm{mg} / \mathrm{d})$ were also given for 4 weeks among all

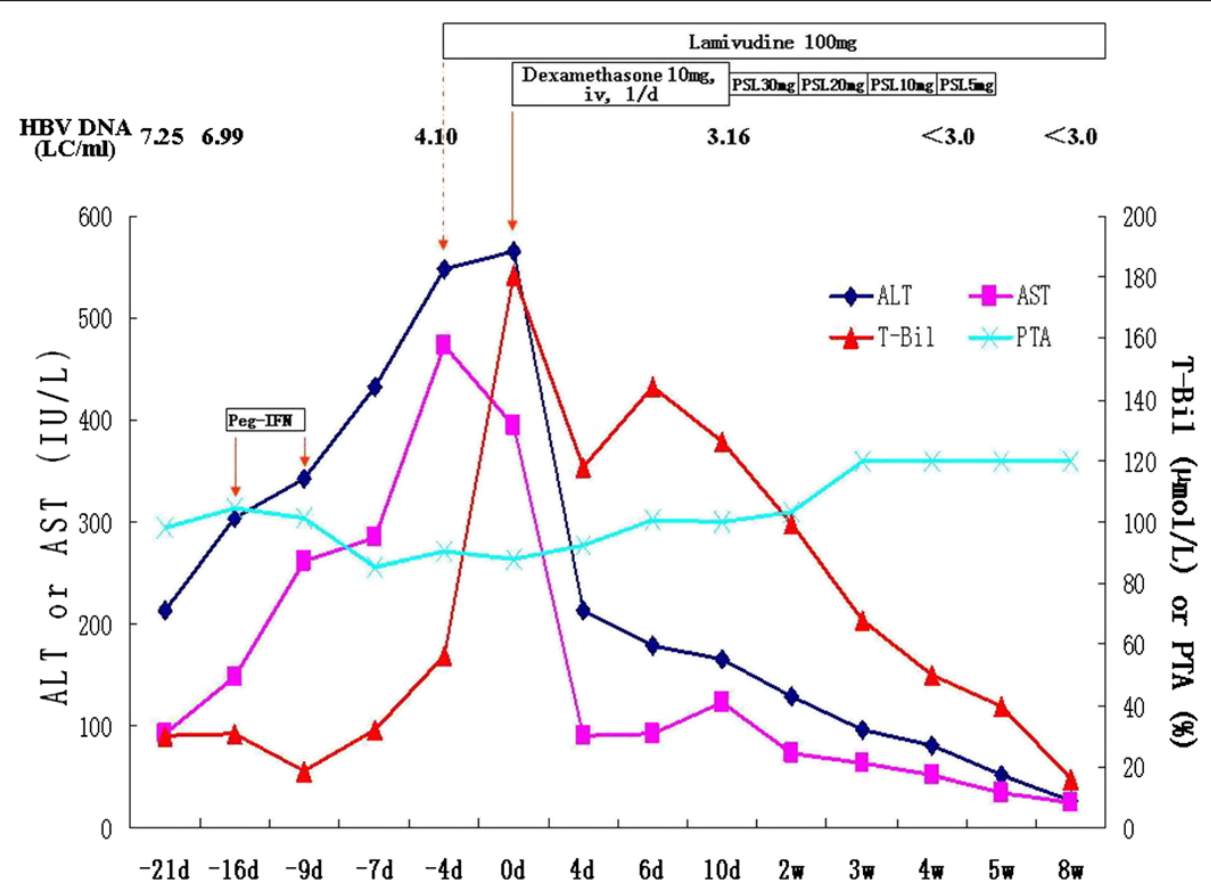

Figure 3 Clinical course of case 3. Peg-IFN: pegylated interferon-a2a; LC/ml: Log $_{10}$ copies/ml; PSL: prednisolone; ALT: alanine aminotransferase; AST: asparate aminotransferase; T-Bil: total bilirubin; PTA: prothrombin activity. 
three patients after being diagnosed to be ACLF or pre-ACLF.

\section{Consent section}

The written informed consents were obtained from three patients before Pegasys and corticosteroid were administrated. Written informed consent was obtained from three patients for publication of this case report and any accompanying images. A copy of the written consent is available for review by the Editor-in-Chief of this journal."

\section{Discussion}

IFN- $\alpha$ is able to increase the expression of HLA-I antigens on hepatocytes, which attracts $\mathrm{T}$ lymphocytes, with subsequent cytolytic and noncytolytic viral inactivation [7]. Acute exacerbation or ALT flare was usually observed among patients with chronic hepatitis B during IFN- $\alpha$ treatment, and appeared to predict a successful outcome [2]. However, patients with severe acute exacerbation tend to have a higher risk for progression to ACLF with extremely high mortality [5,6]. Severe acute exacerbation or ACLF induced by standard interferon- $\alpha$ therapy had been reported to occur in few patients with chronic hepatitis B $[3,4]$. Pegylated interferon- $\alpha$ has been used to treat chronic hepatitis $\mathrm{B}$ and $\mathrm{C}$ for more than five years. Liver failure induced by pegylated interferon$\alpha$ therapy had been reported in chronic hepatitis $C$ [8]. But, no report showed that pegylated interferon- $\alpha$ therapy was able to induce severe acute exacerbation of chronic hepatitis B. Although severe acute exacerbation or ACLF during pegylated interferon- $\alpha$ is rarely occurred, but much attention should be paid by practicing physicians who take care of patients with chronic hepatitis B,because it is a life- threatening adverse effect. In the present cases, severe acute exacerbation of chronic hepatitis B occurred in two patients at the second week of Pegasys treatment, and in one patient at the eighth week of treatment.

$\mathrm{Up}$ to $30 \%$ of patients with chronic hepatitis B, included both $\mathrm{HBeAg}$ - positive and -negative patients, experience spontaneous reactivation of hepatitis every year. Severe acute exacerbation or ACLF may occur among a proportion of patients, and be associated with spontaneous HBe seroconversion[5]. However, we think that severe acute exacerbation or ACLF occurred in three patients described here, is closely associated with Pegasys therapy, but is not a progressive course of underlying hepatitis activity, based on the following reasons. Firstly, case 1 did not spontaneously progress into severe acute exacerbation or ACLF during one month follow-up without antiviral treatment, but progressed into ACLF after two-weeks treatment with Pegasys. Secondly, HBe seroconversion in case 1 was observed after
64 weeks of LAM treatment or severe acute exacerbation being diagnosed, indicated that severe acute exacerbation in case 1 was not associated with spontaneous seroconversion of HBeAg. Thirdly, only mild ALT elevation and normal T-Bil levels were observed in case 2 at the onset and after three weeks of Pegasys treatment. Forthly, severe acute exacerbation was not observed in case 3 during twelve days follow-up from five days of pretreatment to the end of first week Pegasys treatment, but was observed after the five days of second week Pegasys treatment. Additionally, no superinfection by HAV, HCV, HDV, HEV, CMV, EBV and HIV was detected in three patients. Autoimmune hepatitis (AIH), alcoholism and drugs other than Pegasys were also excluded in three patients.

The mortality is very high once ACLF or pre-ACLF develop in patient with severe acute exacerbation of chronic hepatitis B. No evidence showed that antiviral therapy with nucleoside analog (NA) was able to improve short-term mortality in patients with ACLF or pre-ACLF associated HBV infection [5,6]. Case 3 started to receive LAM therapy instead of Pegasys when he was diagnosed to be severe acute exacerbation at $13^{\text {th }}$ day of Pegasys treatment. Interestingly, serum T-Bil was still rapidly increased after 4 days LAM treatment, indicated that short-term antiviral treatment alone was not able to prevent the disease progression once severe acute exacerbation was diagnosed.

However, corticosteroid can rapidly inhibit excessive immune response and inflammatory reaction, and have been confirmed to be effective in treating patients with pre-ACLF and the early stage of ACLF $[6,9]$. Our data also showed that early intervention with corticosteroid and LAM was able to improve the liver function and prognosis of patients with ACLF and pre-ACLF. Our results also indicated that 5-days corticosteroid therapy was enough for rapid responders (the decline extent of serum T-Bil being $\geq 50 \%$ at 5 days), and a tapering-dose corticosteroid therapy was necessary for slow responders $(<50 \%)$.

In summary, Pegasys therapy has a risk of inducing severe acute exacerbation including ACLF and pre-ACLF. Liver function should be tested once a week within 12 weeks of Pegasys therapy in order to find the risk of severe acute exacerbation as early as possible. Importantly, early combined intervention with corticosteroid and lamivudine should be introduced to prevent the disease progression and improve their prognosis once severe acute exacerbation was diagnosed.

\section{Competing interests}

Financial competing interests

1. In the past five years, we did not received any reimbursements, fees, funding, or salary from any organization that may in any way gain or lose financially from the publication of this manuscript. There is not any 

charge).

2. We do not hold any stocks or shares in an organization that may in any way gain or lose financially from the publication of this manuscript, either now or in the future.

3. We are currently applying for any patents relating to the content of the manuscript. We did not receive reimbursements, fees, funding, or salary from any organization that holds or has applied for patents relating to the content of the manuscript.

4. We have not any other financial competing interests.

Non-financial competing interests

There are not any non-financial competing interests (political, personal, religious, ideological, academic, intellectual, commercial or any other) to declare in relation to this manuscript.

\section{Acknowledgments}

The authors thank our colleagues Jing Peng and Li Jiang for assistance in collection of clinical data.

This study was supported by Chinese National Natural Science Foundation grants 30872229

\section{Authors'contributions}

Qing Mao collected the clinical data of one patient and drafted the manuscript, Hui-Yan Zhang collected the clinical data of one patient, JianPing You collected the clinical data of one patient. Xu-Qing Zhang conceived of the study, participated in its design and performed the statistical analysis. All authors read and approved the final manuscript.

Received: 30 December 2011 Accepted: 24 July 2012

Published: 24 July 2012

\section{References}

1. Chan HL, Sung JJ: Hepatocellular carcinoma and hepatitis B virus. Semin Liver Dis 2006, 26:153-161.

2. Akdogan M, Senturk H, Mert A, Tabak F, Ozbay G: Acute exacerbation during interferon alfa treatment of chronic hepatitis $B$ : frequency and relation to serum $\beta-2$ microglobulin levels. J Gastroenterol 2003, 38:465-470

3. Durand JM, Kaplanski G, Portal I, Scheiner C, Berland Y, Soubeyrand J: Liver failure due to recombinant alpha interferon. Lancet 1991, 338:1268-1269.

4. Laskus T, Radkowski M, Slusarczyk J, Cianciara J: Severe exacerbation of chronic active hepatitis B during interferon alpha therapy. Digestion 1992, 52:61-64.

5. Wong S, Chan Y: Severe acute exacerbation of chronic hepatitis B: a unique presentation of a common disease. J Gastroentero and Hepatol 2009, 24:1179-1186.

6. Zhang XQ, Jiang L, You JP, Liu YY, Peng J, Zhang HY, et al: Efficacy of short-term dexamethasone therapy in acute-on-chronic pre-liver failure. Hepatology Research 2010, 41:46-53.

7. Pignatelli M, Waters J, Brown D, Lever A, Iwarson S, Schaff Z, et al: HLA class I antigens on the hepatocyte membrane during recovery from acute hepatitis $B$ virus infection and during interferon therapy in chronic hepatitis B virus infection. Hepatology 1986, 6:349-353.

8. Kogure T, Ueno Y, Fukushima K, Nagasaki F, Inoue J, Kakazu E, et al: Fulminant hepatic failure in a case of autoimmune hepatitis in hepatitis C during peg-interferon-alpha $2 \mathrm{~b}$ plus ribavirin treatment. World J Gastroenterol 2007, 13:4394-4397.

9. Fujiwara K, Yasui S, Yonemitsu Y, Fukai K, Arai M, et al: Efficacy of combination therapy of antiviral and immunosuppressive drugs for the treatment of severe acute exacerbation of chronic hepatitis B. Gastroenterol 2008, 43:711-719.

doi:10.1186/1743-422X-9-136

Cite this article as: Mao et al: Severe acute exacerbation of chronic hepatitis B during pegylated interferon treatment and early intervention with corticosteroid. Virology Journal 2012 9:136.

\section{Submit your next manuscript to BioMed Central and take full advantage of:}

- Convenient online submission

- Thorough peer review

- No space constraints or color figure charges

- Immediate publication on acceptance

- Inclusion in PubMed, CAS, Scopus and Google Scholar

- Research which is freely available for redistribution

Submit your manuscript at www.biomedcentral.com/submit 